\title{
Michael Hartmann
}

\section{Elitehochschulen - die soziale Selektion ist entscheidend}

Seit die SPD im Rahmen ihrer Innovationsoffensive Anfang des Jahres den Begriff der Elitehochschulen in die bildungspolitische Diskussion eingeführt hat, ist er aus der öffentlichen Debatte nicht mehr wegzudenken. In den Medien ist in den vergangenen Monaten eine Vielzahl von Pro- und (deutlich weniger) Contra-Stimmen zu vernehmen gewesen. Trotz unterschiedlicher Meinungen zum Sinn oder Unsinn von Elitehochschulen im Allgemeinen oder (weit häufiger) nur zu den konkreten Plänen der Bundesregierung im Besonderen ist eines auffällig. So gut wie alle Diskussionsteilnehmer sind sich in einem Punkt einig. Es geht bei den Elitehochschulen um die internationale Wettbewerbsfähigkeit der deutschen Wissenschaft und damit zusammenhängend auch der Wirtschaft. Mit dem Verweis auf die sog. „Leuchttürme“ der Wissenschaft in den USA wie Harvard, Yale oder Princeton wird die Schaffung von Elitehochschulen auch hierzulande als die einzige Möglichkeit angepriesen, in der internationalen Wissenschaftskonkurrenz wieder zur Spitze aufzuschließen. Wenn Deutschland dort mitmischen wolle, bleibe keine Alternative zur Konzentration auf wenige Eliteuniversitäten.

Was demgegenüber so gut wie nie thematisiert wird, das sind die sozialen Folgen dieses Schrittes. Der Zusammenhang zwischen sozialer Herkunft und Bildungskarrieren, der aufgrund der eindeutigen Ergebnisse von PISA, IGLU und des OECD-Bildungsberichts inzwischen öffentlich heftig diskutiert wird, spielt in der Debatte um die Elitehochschulen kaum eine Rolle. Schon ein etwas intensiverer Blick in die USA oder die anderen führenden Industriestaaten wie Frankreich, Großbritannien oder Japan, die allesamt seit Jahrzehnten oder gar Jahrhunderten Elitebildungsinstitutionen kennen, hätte hier aber skeptisch stimmen müssen. Was diese Einrichtungen trotz aller Unterschiede vereint, ist nämlich nicht die Spitzenforschung - sie findet nur an den Eliteuniversitäten der USA und Großbritanniens statt -, sondern die sozial höchst selektive Auswahl und Ausbildung der zukünftigen Eliten des jeweiligen Landes. Das ist ihr gemeinsamer Nenner. Wer über die Pläne für Elitehochschulen ernsthaft diskutieren will, sollte sich dementsprechend in erster Linie damit beschäftigen, welche Konsequenzen sie in sozialer Hinsicht für die deutsche Gesellschaft haben werden. Dieser Frage wird der folgende Artikel nachgehen. Zunächst wird 
er sich näher mit der Realität von Elitebildungsinstitutionen in den anderen führenden Industrieländern befassen, um daran anschließend genauer zu analysieren, welche Bedeutung die Debatte um die Eliteuniversitäten für die Umgestaltung der deutschen Hochschullandschaft wirklich besitzt.

\section{Die Elitebildungsinstitutionen in den führenden Industriestaaten}

Welche Wirkung Elitehochschulen in sozialer Hinsicht haben, das lässt sich in Frankreich mit seinen berühmten Grandes Écoles am deutlichsten erkennen. Sie haben im Kern, wie Bourdieu (2004) immer wieder betont, nur eine einzige Funktion. Sie garantieren die Reproduktion der herrschenden Klasse ${ }^{1}$ qua exklusiver Bildungstitel. Auf der einen Seite bildet der Abschluss einer der führenden Grandes Écoles, vor allem der Verwaltungskaderschmiede ENA, der ingenieurwissenschaftlich ausgerichteten École Polytechnique und der wirtschaftswissenschaftlichen HEC, eine fast unverzichtbare Voraussetzung für die Besetzung der Toppositionen in Frankreich. Auf der anderen Seite sorgen diese drei Hochschulen durch ihre sehr selektiven Aufnahmebedingungen gleichzeitig nicht nur für eine gleichbleibend geringe Zahl von Studierenden (wenige hundert ${ }^{2}$ von insgesamt 2,2 Millionen Studierenden), sondern auch für eine sozial hoch exklusive Rekrutierung. Gerade einmal 8,6\% ihrer Studierenden haben eine sog. "Origine populaire" (Bauern, Arbeiter, untere und mittlere Angestellte und Beamte, selbständige Handwerker und Gewerbetreibende) (Eunat/Thélot 1995: 434ff.).

Obwohl Studiengebühren nur an der HEC erhoben werden und die Auslese durch standardisierte Prüfungen auf den ersten Blick allen die gleichen Erfolgsaussichten verspricht, sorgt der Vorsprung, den die bürgerlichen Familien in kultureller wie in ökonomischer Beziehung aufweisen, für eine äußerst effektive soziale Auslese. Außerdem begünstigt die hohe Selbstrekrutierungsquote in den Lehrkörpern der Grandes Écoles von bis zu 90\% all jene deutlich, die aus denselben gehobenen Gesellschaftsschichten wie die Angehörigen dieser Lehrkörper kommen und daher deren Sprache, Geschmack und Umgangsformen reilen oder zumindest gut kennen. Außerdem sorgen die Bildungseinrichtungen selbst für eine Auswahl bereits „prädisponierter" Akteure. So sind die Rekrutierungsprozeduren von Institutionen wie der ENA so angelegt, dass sie ihr Studierende garantieren, die ,aufgrund ihrer familiären Erziehung bereits über die verlangten Dispositionen verfügten, so dass man sich wie die alten Römer fragen muss, ob sie sich nicht damit begnügten, 'den Fischen das Schwimmen beizubringen"s (Bourdieu 2004: 93).

1 Bourdieu hat den Begriff der herrschenden Klasse seit Ende der 80er Jahre allerdings zugunsten des Begriffs vom Feld der Macht aufgegeben. Zu den Gründen für diesen Schritt siehe Bourdieu (2004), zur Kritil an diesem Verzicht und zur Definition der herrschenden Klasse unter heutigen Bedingungen siehe Hartmann (2004).

2 So haben 2003 nur 116 an der ENA ein Studium begonnen, 380 an der HEC und 400 an der Polytechnique. 
De Saint Martin zitiert in ihrer Analyse des französischen Adels aus Prüfungsberichten der ENA, die die Bedeutung der sozialen Herkunft und des daraus resultierenden Habitus für die Zulassung zum Studium an einer der berühmten Grandes Écoles mehr als deutlich machen. Dort heißt es: „Das Prüfungsgespräch dient nicht zur Überprüfung des Wissensstandes ....[Es ist vielmehr] ein methodischer Versuch, menschliche Qualitäten in einem Alter einzuschätzen, in dem man sich noch nicht allzu sehr verstellen kann. ... Ein Mitglied einer Prüfungskommission sagte einmal: 'Ich versuche, mir vorzustellen, ob ich gerne mit dem Kandidaten zusammenarbeiten würde, dem ich gerade zuhöre, und ob ich ihm vollständig vertrauen würde.' Das Bestreben geht also dahin, den Menschen herauszustellen, und nicht irgendein mit Diplomen und Bücherwissen bestücktes Wesen. Das Eingestehen einer Wissenslücke, eine lockere, aber nicht anmaßende Haltung, die Gabe geistesgegenwärtiger Erwiderungen und geistige Neugier sind ausgezeichnete Eigenschaften " (de Saint Martin 1993: 203). Diese Kriterien ähneln sehr stark denen für Topmanagementpositionen in deutschen Großunternehmen und sorgen hier wie dort für eine weitgehende soziale Schließung zu Gunsten der Kandidaten aus bürgerlichen Kreisen (Hartmann 1996, 2001a, 2002).

Auch in Großbritannien stellen exklusive Bildungsinstitutionen das entscheidende Nadelöhr für den Zugang zu den wichtigen Eliten des Landes dar. Die entscheidende Rolle spielen im Unterschied zu Frankreich allerdings weniger die Universitäten, obwohl es mit Oxford und Cambridge auch hier zwei ausgesprochene Eliteeinrichtungen gibt, sondern eine Reihe sehr renommierter Privatschulen. An der Spitze stehen die so genannten Clarendon Nine, die neun berühmtesten Public Schools ${ }^{3}$, zu denen auch Eton zählt. Sie bilden zusammen mit weiteren 20 Topschulen die „Eton and Rugby Groups“, die insgesamt nur 5 Promille eines Jahrgangs besuchen. Durch die enormen Schulkosten von bis zu 19.100 £, det Höhe eines durchschnittlichen Jahreseinkommen, bleiben diese Schulen der breiten Bevölkerung verschlossen. Wie selektiv die Public Schools sind, zeigt schon ein Blick auf die Gesamtheit der HMCSchools ${ }^{4}$, die im Durchschnitt nur gut halb so teuer wie Eton, Winchester oder Westminster sind. Ihre Schüler kommen seit Jahrzehnten zu ca. 90\% aus dem oberen Fünftel der britischen Gesellschaft und zu zwei Dritteln sogar aus den oberen 5\%. An den berühmten Public Schools wie Eton oder Harrow fällt die soziale Rekrutierung aufgrund der erheblich höheren Kosten und der schärferen Auswahlverfahren noch sehr viel exklusiver aus. Es dominieren dort die Kinder der Upper Class.

An den beiden Eliteuniversitäten Oxford und Cambridge setzt sich, wenn

3 Die ca. 500 Public Schools stellen den angeseheneren Teil der Privatschulen. Sie werden von ungefähr 5\% der britischen Sekundarschüler besucht.

4 HMC ist die Ablürzung für Headmaster's conference, die 1869 gegründete Organisation der Public Schools für Jungen, der vier von fünf Schulen dieses Typs angehören. 
auch weniger krass als an den besten Public Schonis, die snziale Selektion dann fort. Das hat vor allem einen Grund. Die Absolventen der Public Schools besuchen diese beiden Hochschulen zu einem weit überdurchschnittlichen Prozentsatz. Ehemalige Public-School-Schüler stellen heute wie schon in den letzten Jahrzehnten jeden zweiten Studierenden an den beiden Topuniversitäten (Adonis / Pollard 1997: 24f.).

Das Elitebildungssystem in den USA ähnelt dem britischen. Die ebenfalls sehr exklusiven und kostspieligen Privatschulen spielen allerdings nur eine eher untergeordnete Rolle im Ausleseprozess. Sie ermöglichen in der Regel keinen direkten Zugang zu den gesellschaftlichen Führungspositionen, sondern bilden nur eine Vorstufe zu den für die Karriere entscheidenden privaten Eliteuniversitäten wie Harvard, Yale oder Stanford. Dabei sind sie allerdings immer noch sehr erfolgreich. Zwar gehen z.B. nicht mehr wie früher drei Viertel der gut 300 Absolventen von Phillips Exeter nach Harvard, sondern „nur" noch ein knappes Sechstel. Rechnet man aber nur die Zulassungszahlen für die zwei bekanntesten Privatschulen, Phillips Exeter und Andover Exeter, zusammen, so kommt man bereits auf 90 Zulassungen von insgesamt gut 1.600 (Levy 1990: 56f.). Allein diese zwei Schulen, von denen gerade einmal 600 bis 700 von insgesamt über zwei Millionen High-School-Absolventen kommen, stellen damit über 5\% der Erstsemester in Harvard. Insgesamt schneiden die Absolventen der bekannten Privatschulen mit einer Zulassungsquote von knapp $40 \%$ an den Topuniversitäten fast ebenso gut ab wie die Kinder von Ehemaligen mit gut 40\%. Da die Privatuniversitäten zu einem großen Teil von den Spenden wohlhabender Alumni oder der Unternehmen, in denen jene tätig sind, abhängen, werden letztere mit Hilfe von Quoten bewusst bevorzugt, um den Zugang zu diesen Geldquellen zu sichern.

Wie angesichts dieser Quoten schon zu vermuten ist, zeigen die renommierten Eliteuniversitäten eine äußerst selektive soziale Zusammensetzung ihrer Studierenden. Gut vier Fünftel stammen aus den oberen 20\% der US-Gesellschaft, während die untere Hälfte der Bevölkerung kaum vertreten ist. ${ }^{5}$ Verantwortlich dafür sind vor allem drei Gründe. Zunächst erzielen die Kinder aus den gehobenen Klassen und Schichten der Gesellschaft in den Leistungstests bessere Ergebnisse. Sie haben eben von Kindesbeinen an nicht nur durch ihr familiäres Umfeld günstigere Bedingungen, sich intellektuell zu entwickeln, sie genieBen auch die bessere schulische Bildung, sei es einfach wegen ihres Wohnorts, sei es aufgrund des Besuchs teurer Privatschulen.

Zwei andere Punkte sind für die soziale Selektion aber noch wichtiger. Zum einen sind die Kosten eines Studiums an einer der Eliteuniversitäten enorm

5 Vermutlich ist die Zusammensetzung noch selektiver. Da die Angaben der Universitäten, soweit sie überhaupt zu finden sind, bei Einkommen oberhalb von $85.000 \$$ in der Regel keine Differenzierung mehr vornehmen, ist nicht sicher zu sagen, wie hoch der Anteil der oberen $10 \%$ oder $5 \%$ ist 
hoch. Sie belaufen sich mittlcrwcilc auf 35.000 bis $40.000 \$$ pro Jahr für das Undergraduate-Studium und 40.000 bis über $50.000 \$$ für alle weiter gehenden Studiengänge bei einem durchschnittlichen Familieneinkommen von gerade cinmal gut 35.000 \$. Die umfangreichen finanziellen Unterstützungsprogramme an den Hochschulen können die davon ausgehende soziale Selektionswirkung nur abschwächen, weil sie bei fast drei Vierteln der Begünstigten zu einem mehr oder minder großen Teil aus verzinslichen Darlehen bestehen. Man kann so schnell auf eine Gesamtverschuldung von ungefähr 60.000 \$ für ein reines Undergraduate-Studium und $200.000 \$$ für ein Masterstudium kommen (Hartmann 2001b). Die Verschuldung eines durchschnittlichen Studierenden, das heißt unter Einbeziehung auch all derjenigen, die an staatlichen Universitäten oder Colleges mit wesentlich niedrigeren Gebühren von zum Teil nur 2.000 bis $3.000 \$$ studieren, liegt inzwischen bei $30.000 \$$. Das ist mehr als eine Verdoppelung binnen sieben Jahren. Potentielle Bewerber aus der Arbeiterklasse oder den breiten Mittelschichten werden dadurch weit stärker abgeschreckt als die Kinder aus wohlhabenden Familien.

Die für die endgültige Zulassung verantwortlichen Admissions Committees bilden den zweiten für die soziale Selektion entscheidenden Engpass. Man sucht an den Eliteuniversitäten explizit nicht nur nach denjenigen, die die besten Testergebnisse aufzuweisen haben, sondern vor allem nach Persönlichkeiten, die einen „Gewinn“ für die Universität darstellen. Die Persönlichkeit des Kandidaten ist also letztlich ausschlaggebend. So bildet „Leadership“ z.B. für die in Harvard zuständige Direktorin das zentrale Kriterium. Wie Feldman (1988) in ihrer Untersuchung über die Zulassungsprozeduren von Harvard deutlich zeigt, begünstigen solche Maßstäbe eindeutig den Nachwuchs aus den „besseren Kreisen“. Bei der Bewertung der Persönlichkeit schneiden zum Beispiel die Absolventen der Privatschulen deutlich besser ab als die anderen Bewerber, in der Bewertung der geistigen Fähigkeiten durch die Zulassungskommission gibt es dagegen keinerlei Unterschiede. Feldman kommt in ihrer Untersuchung zu dem Schluss, dass das derzeitige Auslesesystem im Großen und Ganzen den gleichen sozialen Effekt hat wie eine direkte Versteigerung der Studienplätze gegen Geld. Würde man bei der Auswahl dagegen das Zufallsprinzip oder ein reines Leistungsprinzip walten lassen, würde der Anteil der Ehemaligenkinder wie der Schüler der bekannten Privatschulen nahezu halbiert (Feldman 1988: 176f.).

In Japan spielt die Todai, die staatliche Kaiserliche Universität von Tokio, für den Weg in die entscheidenden Führungspositionen eine ebenso so große Rolle wie die ENA in Frankreich. Ihr folgen die staatliche Kyodai, die öffentliche Hitotsubashi und die privaten Hochschulen Keio und Waseda. Aufgrund zahlreicher Selektionsmechanismen - so kostet der Besuch der führenden Oberschulen inklusive der obligatorischen Nachhilfe an die 10.000 Euro pro Jahr und die Aufnahmeprüfungen ähneln strukturell denen an ENA oder Poly- 
techuique - stammen die Studierenden an der Todai durchweg aus den oberen 10\% der japanischen Gesellschaft (Cutts 1997: 231).

\section{Elitehochschulen und die soziale Rekrutierung der Eliten}

Wer das Examen an einer dieser Eliteinstitutionen bestanden hat, dem steht eine Karriere in allen wichtigen gesellschaftlichen Bereichen offen. In Frankreich sind die Absolventen der ENA, die Enarques, am erfolgreichsten. Allein zwei der drei Staatspräsidenten und sechs der elf Premierminister seit Pompidou waren dort. Außerdem kommen von den Ministerialdirektoren an der Spitze der „Cabinets ministeriell" $70 \%$ von der ENA ebenso wie jeder zweite Direktor in den anderen Ministerien, zwei von drei Präfekten, einer von zwei Botschaftern und vier der fünf Richter am obersten französischen Verwaltungsgericht. Hier macht sich bemerkbar, dass die Enarques die Positionen in den Grand Corps, den Eliteeinrichtungen der öffentlichen Verwaltung, größtenteils unter sich ausmachen (Bock 1999: 390ff.; Kesler 1997: 25ff.; Suleiman 1997: 39).

In den Topetagen der Wirtschaft sieht es nicht anders aus. Die 100 größten französischen Konzerne werden zu über zwei Dritteln von den Absolventen der ENA, der École Polytechnique oder der HEC geführt. Wie ungeheuer stark der Einfluss einiger weniger Eliteeinrichtungen auf die Besetzung des Topmanagements ist, wird noch deutlicher, richtet man sein Augenmerk auf den Anteil derjenigen Absolventen der berühmten Grandes Écoles, die anschließend auch noch in einem der renommierten Grands Cotps waren. Immerhin 40 der 100 größten Firmen werden von solchen Personen gefüht (Hartmann 2001a: 211ff.). Mit gerade einmal 500 Absolventen pro Jahr, das heißt 0,6 Promille eines Jahrgangs, machen die ENA und die École Polytechnique die Mehrzahl der entscheidenden Spitzenpositionen in der französischen Gesellschaft unter sich aus. Ein Großteil wird sogar von einem noch kleineren Kreis besetzt, den ungefähr 80 Studenten, die nach ihrem Examen dort noch in die Grands Corps gehen. Es sind dies ganze 0,1 Promille eines Jahrgangs.

Japan kommt Frankreich diesbezüglich am nächsten. Die Todai mit ihren gut 15.000 Studierenden - das sind gerade einmal 5 Promille aller japanischen Studierenden - stellt z.B. 10 der 25 Premierminister seit 1945 und jeden dritten Minister im derzeitigen Kabinett Koizumi. Die ca. 300 Personen, denen der Zugang zu den Spitzenstellungen in den wichtigsten Ministerien jährlich offen steht, haben zu zwei Dritteln die Todai besucht, die ca. 70 in den beiden wichtigsten Ministerien sogar zu drei Vierteln, die große Mehrzahl davon die juristische Fakultät mit ihren nur 400 bis 500 Abschlüssen pro Jahr, auf der auch 8 der 14 Richter am obersten Gericht des Landes waren. In der Wirt-

6 Bei den zwei renommiertesten privaten Eliteuniversitäten, Keio und Waseda dürfte das, zumal sie (je nach Studienfach) mit 7.000 bis 10.000 Euro pro Jahr deutlich höhere Gebühren verlangen, nicht anders sein. 
srhaft bietet sich ein ähnliches Bild. Von den Präsidenten der 100 größten japanischen Unternehmen kommen allein 40 von Todai und weitere 22 von den anderen vier (Cutts 1997: 179; Hartmann 2002: 154). Zwei Drittel der Wirtschaftselite waren auf einer der fünf berühmtesten Universitäten, über ein Drittel auf Todai und dort vor allem an einer einzigen Fakultät, der juristischen. Mit ganzen 400 bis 500 Absolventen, das heißt nicht mehr als 0,5 Promille aller Hochschulabsolventen und nur gut 0,2 Promille eines Jahrgangs, stellt diese eine Fakultät ungefähr jeden dritten Topmanager, zwei von drei hohen Ministerialbeamten und vier von 10 Ministerpräsidenten.

Ein Examen von Oxford oder Cambridge und, insgesamt sogar noch stärker, ein Abschluss der berühmten Public Schools öffnet auch in Großbritannien die Türen zu den gesellschaftlichen Spitzenpositionen. Von den Chairmen der 100 größten britischen Unternehmen haben über drei Viertel eine der 27 angesehensten Public Schools mit ihren gut 3.000 Absolventen jährlich besucht, elf allein Eton und weitere vierzehn eine der anderen acht Clarendon Schools. Zudem hat nahezu jeder zweite in Oxford oder Cambridge studiert. Die 25 führenden Banken und Versicherungen werden sogar zu fast $90 \%$ von ehemaligen Schülern einer Public School geführt, zu einem knappen Drittel allein von Etonians, und zu knapp zwei Dritteln von Oxbridge-Absolventen (Hartmann 2001a: 165f.). Auch bei den Regierungschefs haben neun der zwölf Nachkriegspremiers in Oxford oder Cambridge studiert, von den Staatssekretären zwei Drittel. Unter den Public Schools spielt Eton die Hauptrolle. Dort war beispielsweise nicht nur fast jeder vierte Staatsekretär, sondern auch ein Viertel der Generäle und der seit 1997 von der Blair-Regierung neu ernannten hohen Richter (Adonis / Pollard 1997: 47f., 59, 125; Barberis 1996: 105).

In den USA fállt der Einfluss der Eliteeinrichtungen aufgrund der Größe des Landes und seiner föderalen Struktur nicht ganz so hoch aus. Anfang der achtziger Jahre hatte von den Topmanagern der 265 größten US-Konzerne zwar jeder zweite an einer von nur zwölf Topuniversitäten studiert, bei einer Reduzierung auf die 100 größten Unternehmen bleibt die übliche Steigerung dieses Prozentsatzes aber aus. „Nur“ noch vier von zehn CEO dieser Firmen waren an einer der 13 Topuniversitäten, jeder vierte allerdings auf einer von nur vier dieser Universitäten und zwölf allein in Harvard (Dye 1995: 171; Hartmann 2001a: 167). In der Politik bietet sich ein ähnliches Bild. Von den elf Präsidenten seit 1945 haben zwar vier in Yale studiert, aber "nur" noch weitere zwei an einer anderen der Top 20 Universitäten. Seit 1989 machen die Yale-Absolventen das Präsidentenamt allerdings unter sich aus. Bush Vater und Sohn waren ebenso dort wie Bill Clinton. Dasselbe trifft im Übrigen auch auf John Kerry, den Herausforderer bei den jetzigen Wahlen, zu. Al Gore, der unterlegene Kandidat der Demokraten bei der letzten Wahl, war in Harvard.

Aufgrund der sozialen Selektivität der Elitebildungseinrichtungen rekrutieren sich die Eliten dieser vier Länder folgerichtig ganz überwiegend aus dem Bür- 
gertum. Typisch ist Frankreich. Zwwei der drei Staatspräsidenten und vier der elf Ministerpräsidenten stammen aus dem Großbürgertum, den oberen 5 Promille der Gesellschaft, nur ganze drei dagegen aus der Arbeiterschaft und den breiten Mittelschichten, den unteren 96,5\%. Die anderen kommen aus dem übrigen bürgerlichem Milieu?. Unter den Topmanagern sieht es genauso aus. Von den PDG der 100 größten Unternehmen ist fast jeder zweite in einer großbürgerlichen Umgebung aufgewachsen und noch einmal gut $40 \%$ in einer bürgerlichen Familie. Der breiten Bevölkerung entstammt demgegenüber nur jeder neunte. Auch in der Verwaltung bietet sich ein eindeutiges Bild. Die Mitglieder der angesehensten Grands Corps kommen zu zwei Drittel, die übrigen hohen Beamten immerhin noch zu ungefähr der Hälfte aus dem Bürgertum. Mit einem Anteil von fast drei Vierteln liegen dabei jene ganz vorn, die die ENA absolviert haben (Rouban 1999: 79ff.). Der Nachwuchs des Bürgertums bleibt weitgehend unter sich, wenn es um die Besetzung der gesellschaftlichen Spitzenpositionen geht.

Ähnlich sieht es in den anderen drei Ländern aus. So stammt fast jeder zweite Chairman der 100 größten britischen Unternehmen aus der Upper Class, im Finanzsektor sogar drei von vier, und ein weiteres Drittel aus dem restlichen Bürgertum (Hartmann 1997: 9f., 2001: 167f., 2002: 159). Von den letzten sechs Premiers Japans hatten gleich drei wichtige frühere Politiker und zwei weitere große Unternehmer (einer davon zusätzlich noch Provinzgouverneur) zum Vater. Typisch ist der jetzige Ministerpräsident Junichiro Koizumi. Sein Vater war Verteidigungsminister und sein Großvater Postminister. Auch in den USA ist die Upper Class in der Politik seit Ende der 80er Jahre wieder sehr stark vertreten. Sowohl die beiden Bushs als auch Al Gore und John Kerry entstammen ihr. Insgesamt aber dürfte die soziale Rekrutierung der Eliten in Japan und den USA nicht ganz so exklusiv ausfallen wie die ihrer französischen und britischen Pendants. Alles in allem aber bleibt als Resümee, dass die Ausbildung an Eliteinstitutionen in allen vier Ländern mit einer hohen sozialen Selektivität einhergeht und die Herkunft letztlich zum ausschlaggebenden Faktor für die Besetzung von Spitzenpositionen wird.

\section{Die Veränderung der deutschen Hochschullandschaft}

Nimmt man die Pläne der Bundesregierung für die Etablierung von Elitehochschulen genauer unter die Lupe, so wird eines relativ schnell klar. Mit den finanziellen Mitteln, die sie dafür vorgesehen hat, ist es absolut unmöglich, zu Spitzenuniversitäten wie Harvard oder Stanford aufzuschließen. Während letz-

7 Zum Großbürgertum zählen Großuntemehmer, Vorstandsmitglieder, Großgrundbesitzer, Spitzenbeamte und Angehörige der Generalität, zum gehobenen Bürgertum als dem restlichen Teil des Bürgertums größere Unternehmer, leitende Angestellte, höhere Beamte und Offiziere sowie akademische Freiberufler. 
teren Jahresetats von 2 bis 2,5 Mrd. \$ für Studierendenzahlen zwischen 11.000 und 17.000 zur Verfügung stehen, müssen die großen deutschen Hochschulen mit 10-20\% dieser Mittel ein Mehrfaches an Studierenden ausbilden. Die jetzt für die noch auszuwählenden Eliteuniversitäten zusätzlich in Aussicht gestellten 250 Mio. Euro pro Jahr werden an dieser Differenz nichts Nennenswertes ändern. Das ist auch den meisten Befürwortern der Elitehochschulen klar. Ihnen geht es im Kern aber auch um etwas ganz anderes. Der Leiter des einflussreichsten Think Tanks in Deutschland, des vielfach auch als inoffizielles Bildungsministerium bezeichneten Centrums für Hochschulentwicklung (CHE) der Bertelsmann-Stiftung, Detlef Müller-Böling, hat das in einer ersten Stellungnahme Anfang Januar auch sehr schnell deutlich gemacht. In dieser Stellungnahme kritisierte er die Pläne der SPD ob ihrer Realitätsferne, so weit es den Vergleich mit Universitäten wie Harvard oder Princeton angeht, machte gleichzeitig aber auf den entscheidenden positiven Effekt der Debatte aufmerksam. Sie mache deutlich, dass die Fiktion einer Gleichheit aller Universitäten aufgegeben werden müsse, und zeige der Öffentlichkeit außerdem deutlich, welche Veränderungen für die deutschen Hochschulen wirklich nötig und zugleich auch realisierbar seien. Das seien die freie Auswahl der Studierenden durch die Hochschulen, weil nur ,im Wettbewerb um die besten Studierenden“ Spitzenleistungen entstünden, die Abschaffung der Kapazitätsverordnung, die den Universitäten eine bestimmte Anzahl von Studierenden pro Professur vorschreibe, weil sie „dem Gedanken der Differenzierung und Elitenbildung“ widerspreche, und die Einführung von Studiengebühren, weil die Kriterien der Weltliga, in der man mitspielen wolle, das einfach zwingend beinhalteten.

Müller-Böling knüpft mit diesen Forderungen an jene Strukturveränderungen an, die seit geraumer Zeit durch zahlreiche einzelne Maßnahmen an den Hochschulen schon Stück für Stück umgesetzt worden sind und weiter werden. Sie zielen alles in allem in erster Linie auf eine wesentlich stärkere Binnendifferenzierung im Sinne einer Hierarchisierung der Hochschullandschaft ab. Die Diskussion um die sog. Eliteuniversitäten hat diesen schon länger laufenden Prozess der Umstrukturierung seit Jahresbeginn allerdings vereinheitlicht und erheblich beschleunigt. Seine wichtigsten Elemente sind zum einen, da trifft Müller-Böling den Kern der Sache, die freie Auswahl der Studierenden durch die Hochschulen und die Einführung von Studiengebühren, zum anderen, und das ist genauso wichtig, das Ende der relativ gleichmäßigen Verteilung der staatlichen Mittel („Gießkannenprinzip") zugunsten einer an sog Leistungskriterien (wie vor allem der Drittmitteleinwerbung) orientierten Mittelvergabe.

Die freie Auswahl der Studierenden durch die Hochschulen ist ein Ziel, das zahlreiche Hochschulen schon seit längerem auf ihre Fahnen geschrieben haben. Institut für Institut, Fachbereich für Fachbereich führt spezielle Aufnahmeprüfungen ein. Begründet wird das in der Regel mit den hohen Abbrecherquoten, die man so reduzieren wolle. Es solle, so die Argumentation, eben nur 
der cin Studium in diesem speziellen Fach beginnen können, der seine Fähigkeiten und seine Motivation zuvor unter Beweis gestellt habe. Das hört sich vernünftig an. Warum sollen 550 Abiturienten an der Uni München mit dem Anglistikstudium beginnen, wenn 200 es schon innerhalb der ersten zwei Semester wieder beenden. Diese Logik hat, selbst wenn man von der hohen sozialen Selektivität solcher Auswahlverfahren erst einmal absieht, einen entscheidenden Fehler. Die Einführung geht zumeist mit einer deutlichen Reduzierung der Studienplätze Hand in Hand. In der Anglistik an der Uni München haben nur ca. 230 den Test bestanden. Es sind nicht nur die 200 vermutlichen Abbrecher „rausgesiebt" worden, sondern noch weitere 120 Bewerber. Bei den Architekten in Berlin oder den Politikwissenschaftlern in Darmstadt fällt der Rückgang mit 120 statt 345 bzw. 76 statt 306 Anfängern noch deutlicher aus. 60 bis $80 \%$ der früheren Studienplätze bleiben auf der Strecke. Die Gelegenheit wird genutzt, um mit der Überlastung vor Ort Schluss zu machen. So wird die Abbrecherquote zwar gesenkt, aber nur um den Preis einer insgesamt geringeren Absolventenzahl. Selbst wenn bei den Anglisten in München statt wie bisher $40 \%$ in Zukunft niemand mehr vorzeitig aufhören sollte, gibt es dennoch 100 Absolventen weniger. Da die deutschen Hochschulen durchweg völlig überlastet sind, gibt es einen Dominoeffekt. Wenn an einer Uni die Zahl der Anfänger beschränkt wird, frei nach dem Motto „verschon mein Haus, zünd's andere an", wird aus Angst vor weiterer Überlastung über kurz oder lang die nächste Uni folgen.

Eine spürbare Reduzierung der Studienplätze wird dabei vor allem jenen Universitäten gelingen, die sich (wie etwa die Hochschulen in Aachen, Berlin, Heidelberg oder München) aufgrund ihres traditionell guten Rufes und/oder ihres städtischen Umfelds eines überdurchschnittlich hohen Zuspruchs erfreuen kötnnen. Wenn die Abiturienten ihre Studienplätze durch Eignungsprüfungen auf Hochschulebene erwerben müssen, können diese besser ausgestatteten und angeseheneren Universitäten die Kriterien für die Aufnahme kontinuierlich verschärfen. Sollte es zudem noch gelingen, die Kapazitätsverordnung abzuschaffen, wäre es ihnen sogar möglich, exklusive Elitestudiengänge mit einer ganz kleinen Zahl von Studierenden einzurichten, ohne die bisherigen Beschränkungen durch die Vorgaben der Kapazitätsverordnung beachten zu müssen. Dieser Gedanke schwebt vielen Hochschulleitungen vor allem an jenen Universitäten vor, die sich zu Recht als die Gewinner der Umstrukturierung fühlen können. Da gilt in besonderem Maße für die aussichtsreichen Kandidaten im Elitewettbewerb. Wenn der Rektor der Universität Heidelberg Anfang Mai in der New York Times International darüber klagt, dass seine Hochschule aktuell 26.000 Studierende beherbergen müsse, während es in den guten alten Zeiten, als Heidelberg noch in der Weltspitze vertreten war, weniger als 10.000 gewesen seien und dabei die Abkehr vom Gleichheitsideal zugunsten des Eliteprinzips sowie indirekt eine drastische Reduzierung der Stu- 
dierendenzahl fordert, dann wird dettlich, wohin der Weg gehen soll. Fs soll wieder einzeİne Eliteuniversitäten geben, die sich den Problemen der unterfinanzierten Massenhochschulen durch eine deutliche Verringerung und zugleich Selektion der zugelassenen Bewerber entziehen können. „Elite und Masse passen nicht zusammen", so formulierte es in dankenswerter Klarheit der Prorektor von Heidelberg, als er in einem Interview die freie Auswahl der Studierenden durch die Hochschulen zu begründen suchte.

Sollte, wie von den meisten Beobachtern erwartet, das Bundesverfassungsgericht Ende des Jahres das Verbot von Studiengebühren für das Erststudium kippen, könnte dieser Prozess weiter beschleunigt werden. Während in den Wissenschaftsministerien in der Regel noch von 500-1.000 Euro jährlich die Rede ist, spricht die Hochschulrektorenkonferenz bereits von Summen von bis zu 3.000 Euro pro Jahr und einzelne Universitätsleitungen denken bereits laut oder halblaut über fünfstellige Gebühren für bestimmte Studienfächer wie etwa Medizin nach. Die Selektionswirkung solcher Kosten liegt auf der Hand. Sie werden die soziale Auslese noch einmal deutlich verstärken, die von den Auswahlverfahren sowieso schon ausgeht. Jene begünstigen durch ihre Kriterien, wie die Bewerbungsprozeduren an den bekannten Universitäten der anderen großen Industriestaaten klar zeigen, ganz eindeutig die Bewerber aus dem Bürgertum. Die Kandidaten, die aus diesem Milieu stammen und den für die Auswahl zuständigen Personen dementsprechend in ihrem Habitus ähneln ungefähr jede zweite Professur wird vom Nachwuchs des Bürgertums besetzt -, werden folglich auch in Deutschland wesentlich bessere Aussichten haben, an den begehrten Hochschulen aufgenommen zu werden. Manchmal wird der soziale Selektionsgehalt solcher Auswahlgespräche sogar bei oberflächlicher Betrachtung unübersehbar. So stellt das Institut für Politikwissenschaften an der TU Darmstadt Abiturienten als ein wichtiges Bewertungskriterium die Frage: „Welche ausländischen Tageszeitungen lesen Sie?". Was hier offenkundig ist, bleibt allerdings zumeist auf den ersten Blick verschlossen und wird erst bei genauerem Hinsehen erkennbar. Auswahlgespräche selektieren eben nicht nur nach Leistung, sondern auch nach Herkunft, und das nicht nur auf dem Wege der sozialen Bedingtheit schulischer Leistungen, sondern auch ganz unmittelbar durch die von Bourdieu so detailliert geschilderte Affinität zwischen den Kandidaten und den von ihnen ausgewählten Bildungsinstitutionen.

Die geplante Binnendifferetazierung der deutschen Hochschullandschaft setzt neben der freien Auswahl der Studierenden und der Einführung von Studiengebühren allerdings noch eine weitere Veränderung voraus. Die Verteilung der staatlichen Mittel muss stärker als schon bisher auf jene Universitäten konzentriert werden, die nach den in der öffentlichen Diskussion üblichen Kriterien (vor allem Drittmittelanteil) als besonders leistungsstark gelten. Sie wird also all jene Hochschulen begünstigen, die aufgrund ihrer traditionellen Verbindungen zu Wirtschaft und Politik oder ihrer Fächerstruktur (Medizin, In- 
genieur- und Naturwissenschaften) im Vorteil sind und bisher schon über mehr Mittel verfügen konnten. Ihr finanzieller Vorsprung wird durch die neuen Finanzierungsrichtlinien spürbar weiter ausgebaut. Die Kluft zwischen den Hochschulen wird damit erheblich größer. Wie dieser Prozess ablaufen wird, davon gibt die Gründung des bayerischen Elitenetzwerks einen Vorgeschmack. Bei sieben der zehn in diesem Zusammenhang durch Extramittel geförderten Projekte haben die beiden Münchener Universitäten die Federführung, während umgekehrt die Hochschulen in Bamberg und Passau keinen einzigen Euro Fördermittel aus diesem Topf erhalten. Der Teufel scheißt, wie der Volksmund sagt, eben immer auf den größten Haufen.

Die deutsche Hochschullandschaft wird, sollte dieser Weg, wonach derzeit vieles aussieht, weiter beschritten werden, in 10 bis 15 Jahren stark hierarchisiert und sozial sehr viel selektiver sein als bisher. Zwar rekrutieren sich auch heute schon fast zwei Drittel der Studierenden an den deutschen Universitäten aus dem oberen knappen Drittel der Gesellschaft und 40\% sogar aus dem oberen Siebtel ${ }^{8}$, die soziale Selektivität ist verglichen mit den Elitehochschulen der anderen Länder, wo die oberen 10-20\% der Bevölkerung 80-90\% der Studierenden stellen, aber maximal halb so hoch. In Zukunft wird sich das ändern. Es wird dann Universitäten mehrerer Kategorien geben. Die der 1. und der 2. Kategorie werden einen weit überproportionalen Teil der privaten und staatlichen Gelder erhalten und sich bei den Wissenschaftlern wie den Studierenden "die Rosinen rauspicken“ können. Sie werden in weit stärkerem Maße als heute für den Nachwuchs des Bürgertums reserviert sein, während die Masse der Studierenden aus der breiten Bevölkerung mit den anderen Hochschulen vorlieb nehmen muss, die aufgrund fehlender finanzieller wie personeller Ressourcen immer schlechtere Bedingungen bieten werden.

Die von vielen Kritikern der Hochschulreformen befürchtete Privatisierung ${ }^{9}$ stellt meiner Meinung nach für die deutsche Hochschullandschaft auf absehbare Zeit dagegen keine gravierende Bedrohung dar. Das gilt in doppelter Hinsicht. Zum einen ist keine Umwandlung der staatlichen Hochschulen in private zu erwarten. Die jetzt beschlossene Autonomie von Hochschulen wie der TU Darmstadt oder die Etablierung von Stiftungsuniversitäten wie Göttingen erinnern auf den ersten Blick zwar an private US-Universitäten, im Kern aber hängen diese Hochschulen auch weiterhin am Tropf der staatlichen Mittel. So besteht z.B. das Stiftungskapital von Göttingen aus den Grundstü-

8 Angaben aus der 17. Sozialerhebung des Deutschen Studentenwerks und eigene Berechnungen nach den Angaben über die Bildungsbeteiligung der vier dort gebildeten sozialen Herkunftsgruppen und die soziale Zusammensetzung der Studierenden an den Universitäten (Isserstedt et. al. 2004: 118, 138, 472).

9 Mit Privatisierung ist hier eine strukturelle Veränderung der Hochschullandschaft gemeint und nicht die unbestreitbare Privatisierung eines Teils der Kosten durch die geplanten allgemeinen Studiengebühren; denn letzteren käme erst bei Größenordnungen wie in den USA eine strukturelle Wirkung zu. 
cken, auf denen größtenteils die Universitätsgebäude stehen. Ein Vergleich mit den US-Stiftungshochschulen ist also allenfalls sprachlich gegeben.

Zum anderen dürften die privaten Hochschulen in den nächsten 10-20 Jahren kaum in der Lage sein, zu einer ernsthaften Konkurrenz für die führenden staatlichen Universitäten zu werden. Wie nicht zuletzt die jüngsten Pleiten privater Hochschulen in Stuttgart und Kassel, die chronischen Finanzproblem der einzigen privaten Volluniversitäten in Witten-Herdecke und Bremen sowie der für die deutsche Wirtschaft mehr als peinliche Gründungsprozess der ESMT in Berlin zeigen, ist das Engagement der Kreise, die öffentlich immer wieder nach solchen Privatuniversitäten rufen, letztlich bei weitem nicht ausreichend, wenn es um die tatsächliche Umsetzung solcher Pläne geht. ${ }^{10}$ Ein massives Engagement privater Sponsoren ist aber die Mindestvoraussetzung dafür, dass die privaten Hochschulen überhaupt eine Chance in dieser Konkurrenz haben. Die staatlichen Universitäten der „ersten Liga“ werden in wenigen Jahren vor allem aufgrund der geänderten Mittelverteilung und der restriktiven Auswahlverfahren nämlich deutlich günstigere Bedingungen bieten können als bisher. Dazu kommt dann bei Universitäten wie etwa Freiburg, Heidelberg oder München noch der entscheidende Vorteil, dass ihr traditionell guter Ruf (auch international) im Rahmen einer stark hierarchisierten Hochschullandschaft gerade auf dem Arbeitsmarkt wie ein Markenzeichen wirkt. Dem können die privaten Hochschulen in absehbarer Zeit nichts Ernsthaftes entgegenstellen. ${ }^{11}$ Auch die Einführung von Studiengebühren an den staatlichen Hochschulen wird die Chancen der Privathochschulen nicht nennenswert verbessern, weil nur die Spitzenuniversitäten Summen verlangen werden, die denen der privaten Konkurrenz wenigstens halbwegs entsprechen, dafür dann aber auch die genannten Vorzüge bieten können.

\section{Welche Kräfte stehen hinter der Forderung nach Elitehochschulen?}

Die Forderung nach Elitehochschulen wird von unterschiedlichen Motiven gespeist. In bildungspolitischer Hinsicht verspricht sie die Lösung von zwei zentralen Problemen. Auf der einen Seite erhofft man sich vor allem auf Sei-

10 Im beruflichen Bildungswesen bildet die Privatisierung demgegenüber eine reale Gefahr. Hier gibt es anders als im Hochschulsektor eine lange Tradition privater Bildungsinstitutionen ( $z$. B. in der Ausbildung von Technikern oder in der Weiterbildung), die eine wesentliche Grundlage für weitere Privatisierungsschritte bietet. Ein Beispiele ist die geplante Gründung der "Gesellschaft für Bildungsinfrastruktur mit beschränkter Haftung" in Bremen, die zwar das gesamte Bildungswesen privatisieren soll, zunächst aber faktisch nur die Berufsschulen im Visier hat.

11 W'e schwer es für private Hochschulen ist, in einem System mit staatlichen Spizzenhochschulen deren auf ihrem traditionellen Image beruhenden Vorsprung aufzuholen, zeigt das Beispiel der 1881 gegründeten HEC. Obwohl diese von der Pariser Handelskammer getragene Grande École eine massive Unterstützung seitens der Pariser Geschäftswelt und zahlreicher Großunternehmen erfuhr, brauchte sie fast ein Jahrhundert, um zu den führenden staatlichen Grandes Écoles aufzuschließen (Bourdieu 2004: 238ff.). 
Len der Wirtschaft, auf diese Weisc dic Lcistungsfähigkeit der deutschen Universitäten in der Spitze zu verbessern, ohne dafür umfangreichere staatliche Mittel aufwenden zu müssen. Wollte man die Unterfinanzierung der deutschen Hochschulen insgesamt ernsthaft angehen, müsste man sich fast zwangsläufig mit der Frage höherer Steuern befassen. Das ist jedoch derzeit so ziemlich das letzte, was die Unternehmen sich wünschen, fordern sie doch ganz im Gegenteil ständig noch weitere steuerliche Entlastungen. Eine stärkere Konzentration der Mittel auf einige wenige Universitäten scheint hier eine Lösung zu bieten. Außerdem kann man mit den Studiengebühren gleichzeitig auch verstärkt auf eine Eigenbeteiligung der Studierenden und ihrer Eltern setzen und damit die Privatisierung der Bildungskosten vorantreiben. Damit wäre der Widerspruch zwischen Steuerentlastungen und Bildungsinvestitionen zumindest teilweise entschärft.

Auf der anderen Seite stehen auch große Teile der akademisch ausgebildeten Berufsgruppen hinter den Plänen, weil sie in der Hierarchisierung der Hochschullandschaft die Möglichkeit sehen, die Chancen des eigenen Nachwuchses in der härter werdenden Konkurrenz spürbar zu verbessern. Das gilt gleich in doppelter Hinsicht. Zum einen erhoffen sie sich dadurch trotz der weitergehenden Sparmaßnahmen an den Hochschulen eine qualitativ hochwertige Ausbildung der eigenen. Kinder - dass die es auf die in der Hierarchie höher angesiedelten und dementsprechend besser ausgestatteten Universitäten schaffen, wird stillschweigend vorausgesetzt -, zum anderen glauben sie, die eigenen Sprösslinge durch den Besuch der dann im Ranking höher eingestuften Hochschulen vor der zunehmenden Arbeitslosigkeit unter Hochschulabsolventen bewahren und ihnen die eigene soziale Position qua Bildungsabschluss vererben zu können. $\mathrm{Ob}$ all diese Hoffnungen sich erfüllen, bleibt abzuwarten. Ganz unrealistisch sind die Annahmen jedenfalls nicht. Denn auch wenn keine deutsche Hochschule auf absehbare Zeit die Funktion der Grandes Écoles, der Iry League Universitäten, von Oxbridge oder Todai wahrnehmen wird ${ }^{12}$, so kann die Hierarchisierung der Universitäten eine Ebene darunter die Verteilung der beruflichen Karrierechancen doch erheblich beeinflussen. Wenn beispielsweise Heidelberg oder München, wie zu vermuten, zu den Hochschulen der 1. Kategorie zählen werden, dann werden ihre Absolventen bei der Berufssuche und im weiteren Verlauf ihres Berufslebens davon spürbar profitieren. Ist es heute für die Karriere ziemlich gleichgültig, auf welcher Universität jemand sein Examen gemacht hat, so wird das in 10 Jahren ganz anders sein. Ein Abschluss in Heidelberg oder an der RWTH Aachen eröffnet dann ganz andere Möglichkeiten als einer in Oldenburg oder Duisburg/Essen. Da die Zulassung an den „besseren“" Hochschulen, wie beschrieben, sozial selektiv

12 Dafür sorgen in Deutschland zumindest in der Wirtschaft andere Mechanismen (Hartmann 1996, 2002). 
ausfallen wird, hat die Hoffnung, die sozialen Status auf diese Art und Weise vererben zu können, durchaus eine reale Grundlage.

Für die zügige Umsetzung der Umstrukturierungspläne im Hochschulbereich gibt es aber noch einen weiteren Grund, die fast uneingeschränkte Unterstützung durch die maßgeblichen Eliten dieses Landes. Aus ihrer Sicht passt die Forderung nach Elitebildung und Differenzierung genau in eine Zeit, in der die wachsende Spaltung der Gesellschaft nach politischer Legitimierung verlangt. Wenn es unter Hinweis auf die für den Standort Deutschland unverzichtbaren „Leuchttürme" der Wissenschaft gelingen sollte, die Notwendigkeit von Elitebildung gesellschaftlich zu etablieren, dann dürfte der breiten Öffentlichkeit auch die Teilung der Gesellschaft in oben und unten, Elite und Masse leichter zu vermitteln sein, so die Überlegung.

\section{Literatur}

Adonis, A. / Pollard, S. (1997): A Class Act. The Myth of Britain's Classless Society. London.

Barberis, P. (1996): The Elite of the Elite. Ipswich.

Bock, H. M. (1999): Republikanischer Elitismus und technokratische Herrschaft, in: Christadler, M./ Utterwedde, H. (Hg.): Länderbericht Frankreich. Opladen, 381-403.

Bourdieu, P. (2004): Der Staatsadel. Konstanz.

Cutts, R. L. (1997): An Empire of Schools. Japan's Universities and the Moulding of a National Elite. Armonk.

Dye, T. R. (1995): Who's Running America? The Clinton Years. Englewood Cliffs.

Euriat, M. / Thelot, C. (1995): Le recrutement social de l'elite scolaire en France, in: Revue française de sociologie, 36, 403-438.

Feldman, P. H. (1988): Recruiting an Elite. New York

Hartmann, M. (1996): Topmanager - Die Rekrutierung einer Elite. Frankfurt a. M.

Hartmann, M. (2001a): Klassenspezifischer Habitus oder exklusive Bildungstitel als Selektionskriterium? Die Besetzung von Spitzenpositionen in der Wirtschaft, in: Krais, B. (Hg.): An der Spitze. Deutsche Eliten im sozialen Wandel. Konstanz, 157-215.

Hartmann, M. (2001b): Bildung und andere Privilegien, in: Kursbuch 143: Der Neid. Berlin, 39-53.

Hartmann, M. (2002): Der Mythos von den Leistungseliten. Spitzenkarrieren und soziale Herkunft in Wirtschaft, Politik, Justiz und Wissenschaft. Frankfurt a. M.

Hartmann, M. (2004): Elitesoziologie. Eine Einführung. Frankfurt a. M.

Isserstedt, W. / Middendorff, E. / Weber, S. / Wolter, A. / Schnitzer, K. (2004): Die wirtschaftliche und soziale Lage der Studierenden in der Bundesrepublik Deutschland 2003. 17. Sozialerhebung des Deutschen Studentenwerks. Bonn: Bundesministerium für Bildung und Forschung.

Kesler, J.-F. (1997): L'Enarchie n'existe pas, in: Pouvoirs. Revue Francaise d'etudes constitutionelles et politiques, 80, 23-41.

Levy, A. H. (1990): Elite Education and the Private School. Excellence and Arrogance at Phillips Exeter Academy. Lewiston.

Mougel, F.-C. (1990): Élites et système de pouvoir en Grande-Bretagne 1945-1987. Bordeaux.

Rohlen, T. P. (1983): Japan's High Schools. Berkeley.

Rouban, L. (1999): The Senior Civil Service in France, in: Page, Edward C. / Wright, Vincent (Eds.): Bureaucratic Elites in Western European States. Oxford, 65-89.

Saint Martin, M. de (1993): Der Adel. Soziologie eines Standes. Konstanz.

Scott, J. (1991): Who Rules Britain? Cambridge.

Suleiman, E. (1997): Les élites de l'administration et de la politique dans la France de la V. République: Homogénéité, puissance, permanence, in: Suleiman, E. / Mendras, H. (Eds.): Le recrutement des élites en Europe. Paris, 19-47 